\title{
Adjustment of the Fuzzy Logic controller parameters of the energy management strategy of a grid-tied domestic electro-thermal microgrid using the Cuckoo search algorithm
}

\author{
Diego Arcos-Aviles \\ Department of Electrical \\ and Electronics \\ Engineering \\ Universidad de las Fuerzas \\ Armadas ESPE \\ Sangolquí, Ecuador \\ dgarcos@espe.edu.ec \\ Julio Pascual \\ Department of Electrical \\ and Electronics \\ Engineering \\ Universidad Pública de \\ Navarra \\ Pamplona, Spain \\ juliomaria.pascual@unavarra.es
}

\author{
Gabriel García-Gutiérrez \\ Department of Electrical \\ and Electronics \\ Engineering \\ Universidad de las Fuerzas \\ Armadas ESPE \\ Sangolquí, Ecuador \\ gmgarcia94@gmail.com
}

Paúl Ayala
Department of Electrical
and Electronics
Engineering
Universidad de las Fuerzas
Armadas ESPE
Sangolquí, Ecuador
jpayala@espe.edu.ec

\author{
Francesc Guinjoan \\ Department of Electronics \\ Engineering \\ Universitat Politècnica de \\ Catalunya-Barcelona \\ TECH \\ Barcelona, Spain \\ francesc.guinjoan@upc.edu \\ Luis Marroyo \\ Department of Electrical \\ and Electronics \\ Engineering \\ Universidad Pública de \\ Navarra \\ Pamplona, Spain \\ luisma@unavarra.es
}

\author{
Enrique V. Carrera \\ Department of Electrical \\ and Electronics \\ Engineering \\ Universidad de las Fuerzas \\ Armadas ESPE \\ Sangolquí, Ecuador \\ evcarrera@espe.edu.ec \\ Emilia Motoasca \\ Department of Electrical \\ Engineering \\ Faculty of Engineering \\ Technology-TC Ghent \\ KU Leuven \\ Ghent, Belgium \\ emilia.motoasca@kuleuven.be
}

\begin{abstract}
During the last century, population growth, together with economic development, has considerably increased the energy demand and, although renewable energies are becoming an alternative, still total energy supply is mainly non-renewable, causing well-known negative effects such as pollution and global warming. On the other hand, technological advances have allowed the development of increasingly efficient distributed generation systems and the emergence of microgrids, whose studies have been focused on architecture, elements, and objectives of the associated energy management strategies. In this regard, energy management strategies based on a Fuzzy Logic controller have been developed for electro-thermal microgrids where parameter optimization has been carried out through heuristic procedures of trial and error with acceptable results but involving a high computational cost. To solve the aforementioned drawbacks, in the present work the use of Cuckoo Search optimization nature-inspired algorithm that allows the adjustment of Fuzzy Logic controller parameters and ensures a higher quality of energy management is proposed. Obtained results show encouraging outcomes for the use of these meta-heuristic optimization algorithms.
\end{abstract}

Keywords-energy management, Cuckoo search, Fuzzy Logic control, parameter optimization

\section{INTRODUCTION}

The growth of energy demand, derived from population growth and economic development, has given way to an increase in energy consumption in recent decades. According to information published by the International Energy Agency (IEA), the World total primary energy supply (TPES) (i.e., energy available in nature, before being converted or transformed), obtained mainly from sources such as: coal, oil, natural gas, nuclear, hydro, biofuels and biomass, others (geothermal, solar, heat, wind); has increased from 6,101 million tonnes of oil equivalent (Mtoe) in 1973 to 13,761
Mtoe in 2016 [1], which represents an increase in energy consumption of $125 \%$ in this period.

The increase in energy consumption over the years has had a negative impact on the concentration of greenhouse gases (GHG) in the atmosphere [2], so that, many countries in the world have focused their efforts on improving energy efficiency and increasing the production of clean energy. As a result of these initiatives, there has been a substantial increase in the use of renewable energies sources (RES) in the last decades, where photovoltaic (PV) and wind energy (WT) have represented the highest growth worldwide.

The benefits that RESs have shown in recent years, such as reducing the emission of GHG [3], have supported the development and performance of distributed generation (DG) systems. In this context, new problems have arisen when trying to combine renewable sources together with traditional sources of energy, some of these can be: unexpected fluctuations in the response of RES which can affect the voltage and frequency of the network, power electronics requirements, and different control and dispatch methods.

In order to solve the aforementioned drawbacks, the term microgrid (MG) is born; which was initially defined as a set of micro-sources, charges, and storage systems that operate with a single controllable system that can respond to signals from a central controller [4]. This concept has been discussed by several researchers over the past decade [5]-[9]. MGs are currently defined as a low-voltage distribution network consisting of loads, distributed generation elements, and energy storage systems (ESS) that are connected to the main supply network at a single point of common coupling (PCC), with an associated energy management system (EMS) that allows them to operate reliably, safely, and economically [10], [11]. 
According to the microgrid architecture, the EMS is in charge of controlling the power flux within the different MG's elements with the aim of achieving a set of predefined objectives such as minimizing operating cost, maximizing the $\mathrm{MG}$ revenues or minimizing the fluctuations and ramprates in the power exchanged with the grid [12]-[17].

Considering this last objective, several EMSs have been designed in the last years [18]-[22], obtaining satisfactory results in the minimization of the grid power profile. These studies share a common Fuzzy Logic Control (FLC) block, which is designed according to the heuristic knowledge about the proper MG's operation. However, the FLC design involves the selection of several parameters such as: type of membership functions (MF), number of MFs per input/output, mapping (i.e., location of each MF within the established range for each input/output), and rule base (RB); which makes it difficult to select a set of parameters that minimize the grid power profile.

The adjustment procedure of the FLC parameters of the EMSs described in [18]-[22] consists of three steps which are described in [23]. Although the obtained results, through the aforementioned process, were highly satisfactory, there is a need to have a robust and powerful adjustment (optimization) algorithm capable of tuning the FLC parameters in a fast and efficient manner, so that it improves the behavior of the EMS, and consequently, the quality of the grid power profile of a residential MG.

During the last decades, meta-heuristic nature-inspired algorithms have been given special attention due to their great capability in solving optimization problems in a wide range of applications including the operation and control of electric power systems [24]-[26], where Cuckoo search (CS) algorithm has shown superiority over other algorithms in solving optimization problems in terms of better-obtained results and faster convergence speed [27], robustness and precision of obtained results [28], and its well-balanced intensification/diversification search strategies [29]-[32].

Therefore, this article proposes a new methodology for adjusting the FLC parameters of the EMS of a residential grid-connected electro-thermal microgrid by means of the CS algorithm. A global cost function, including all the criteria used to evaluate the quality of the grid power profile, defined in [16], [18], [19], is formulated with the aim of constituting the objective function of the optimization algorithm. A simulation comparison with the fuzzy-based EMS presented in [20] will demonstrate the improved behavior of the EMS after the FLC is subjected to the proposed parameter adjustment process.

This paper is organized as follows. Section II presents the electro-thermal microgrid under analysis and describes the criteria associated with the grid power profile quality. Section III presents the FLC parameter adjustment using CS algorithm. Section IV presents the simulation and comparison results. Finally, Section V presents the main conclusion of this work.

\section{MicrogiRd ARCHITECTURE AND ENERGY MANAGEMENT STRATEGY}

This study uses the same microgrid architecture and the EMS that the one presented in [20]. However, for the paper completeness, a short description of this architecture and the EMS associated with it is given next.

\section{A. Microgrid architecture}

The MG architecture includes a Hybrid RES comprising of a PV generator of $6 \mathrm{kWp}$ and a WT generator of $6 \mathrm{~kW}$, a battery ESS of $72 \mathrm{kWh}$ capacity ( $36 \mathrm{kWh}$ of useful battery capacity), domestic load demand $\left(P_{L O A D}\right)$ of $7 \mathrm{~kW}$, and a domestic hot water system (DHW) which includes an electric water heater $(\mathrm{EWH})$ of $2 \mathrm{~kW}$, a solar thermal collector of $2 \mathrm{~kW}$, thermal demand equivalent to $2 \mathrm{~kW}\left(Q_{D H W}\right)$, and a hot water tank of 800 liters capacity, as shown in Fig. 1.

In Fig. $1 P_{G E N}$ represents the total renewable power generation, $P_{P V}$ and $P_{W T}$ are the PV and WT power, respectively, $P_{B A T}$ represents the battery power which is positive for discharging process and is negative otherwise, $P_{G R I D}$ is the power exchanged with the gird which is positive when the utility grid injects power to the MG and is negative otherwise, $P_{W H, E}$ is the power required by the electric water heater to meet the required thermal demand, $Q_{S T, T}$ is the thermal storage capacity of the hot water tank, $Q_{L O S S, T}$ represents the thermal losses in the hot water tank, $Q_{S C, T}$ is the rate of energy collected from the solar thermal collectors, and $Q_{W H, T}$ is the rate of energy transferred from the EWH.

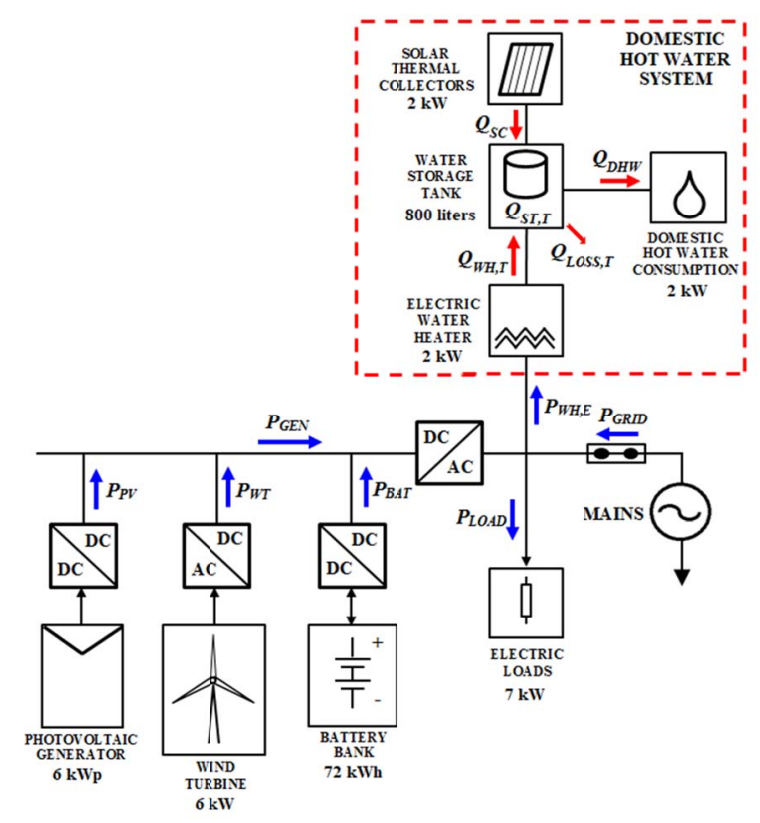

Fig. 1. Electro-thermal microgird architecture [20]. (C)2018 IEEE, Reprinted, with permission form D. Arcos-Aviles et al., "Fuzzy-based energy management of a residential electro-thermal microgrid based on power forecasting," in IECON 2018 - 44th Annual Conference of the IEEE Industrial Electronics Society, Washington, DC, USA, Oct. 2018, pp. 1824-1829.

According to the configuration shown in Fig. 1, the generation power, the MG net power, $P_{L G}$, and the battery power are defined as follows:

$$
\begin{gathered}
P_{G E N}(n)=P_{P V}(n)+P_{W T}(n), \\
P_{L G}(n)=P_{L O A D}(n)+P_{W H, E}(n)-P_{G E N}(n), \\
P_{B A T}(n)=P_{L G}(n)-P_{G R I D}(n) .
\end{gathered}
$$

\section{B. Energy management strategy}

The main goal of the EMS presented in [20] is smoothing the grid power profile through the use of the energy stored in the ESS for covering part of the energy required by the EWH for keeping the water temperature in the tank between 
established limits. The block diagram of this EMS is depicted in Fig. 2, defining the grid power as the sum of three components, as follows:

$$
P_{G R I D}(n)=P_{C T R}^{*}(n)+P_{S O C}(n)+P_{F L C}(n),
$$

where $P_{C T R}^{*}(n)$ is the central moving average (CMA) filter output which establishes the MG average power profile, $P_{S O C}(n)$ is the battery control loop output which is used to keep the battery state-of-charge (SOC) center close to the $75 \%$ of the rated battery capacity, and $P_{F L C}(n)$ is the FLC output. A complete description of each block shown in Fig. 2 can be found in [20].

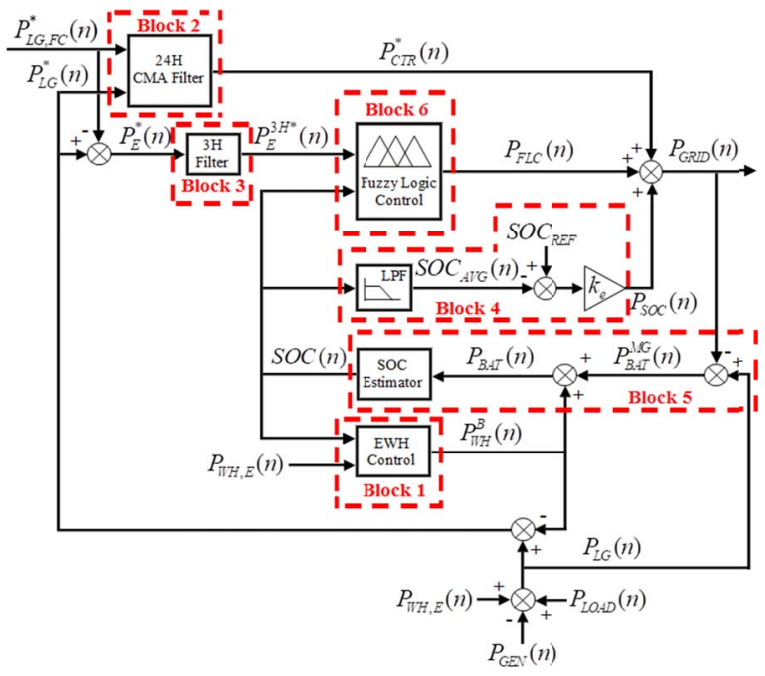

Fig. 2. Energy management strategy block diagram [20]. (C2018 IEEE, Reprinted, with permission form D. Arcos-Aviles et al., "Fuzzy-based energy management of a residential electro-thermal microgrid based on power forecasting," in IECON 2018 - 44th Annual Conference of the IEEE Industrial Electronics Society, Washington, DC, USA, Oct. 2018, pp. 1824-1829.

\section{Grid power profile quality criteria}

The quality criteria are used to quantify the improvement of the grid power profile achieved by an EMS where a lower criteria value implies an improved grid power profile. These criteria have been defined in [15], [16], [18], [19], where a set of 6 quality criteria has been considered for building the optimization cost function. These criteria are: maximum power delivered by the grid in one year $\left(P_{G, M A X}\right)$, maximum power fed into the grid $\left(P_{G, M I N}\right)$, maximum power derivative $(M P D)$, average power derivative $(A P D)$, power variation range $(P V R)$, and power profile variability $(P P V)$, defined as follows:

$$
\begin{gathered}
P_{G, M A X}=\max \left(P_{G R I D}\right), \\
P_{G, M I N}=\min \left(P_{G R I D}\right), \\
M P D=\max \left(\left|\dot{P}_{G R I D}\right|\right), \\
A P D=\frac{1}{N} \sum_{n=1}^{N}\left|\dot{P}_{G R I D}(n)\right|, \\
\dot{P}_{G R I D}(n)=\left[P_{G R I D}(n)-P_{G R I D}(n-1)\right] / T_{S}, \\
P V R=\frac{P_{G, M A X}-P_{G, M I N}}{P_{L G, M A X}-P_{L G, M I N}},
\end{gathered}
$$

$$
P P V=\sqrt{\sum_{f=f_{i}}^{f_{f}}\left(P_{G R I D, f}\right)^{2}} / P_{D C},
$$

where $\dot{P}_{\text {GRID }}$ is the grid power profile ramp-rate (i.e., the slope of two consecutive sampiles of the grid power profile), $N$ the number of samples in one year, $P_{G R I D, f}$ is grid power harmonic at $f$ frequency, $f_{i}$ and $f_{f}$ are the initial and final frequencies, respectively, and $P_{D C}$ is the yearly power average value. Note that $f_{i}=1.65 \times 10^{-6} \mathrm{~Hz}$ and $f_{f}=5.55 \times 10^{-4} \mathrm{~Hz}$ to evaluate frequencies above one week or less variation periods [15], [16], [18], [19].

Based on the aforementioned quality criteria, which are the objectives functions, the optimization cost function, i.e., fitness function, is defined as follows:

$$
\begin{gathered}
\text { fitness }=w \cdot F_{1}+(1-w) \cdot F_{2}, \\
F_{1}=\left(\frac{P_{G, M A X}}{P_{G, M A X}^{R E F}}+\frac{P_{G, M I N}}{P_{G, M I N}^{R E F}}+\frac{M P D}{M P D_{R E F}}\right), \\
F_{2}=\left(\frac{A P D}{A P D_{R E F}}+\frac{P V R}{P V R_{R E F}}+\frac{P P V}{P P V_{R E F}}\right),
\end{gathered}
$$

where $w$ is a real number weighting the two components of the cost function and is fixed by the user to prioritize either $F_{1}$ or $F_{2}$. It can be pointed out that to limit the variation range of $F_{1}$ and $F_{2}$ each quality criteria is normalized with respect to a reference value which is intended to be minimized in the optimization process [33].

\section{FUZZY LOGIC PARAMETERS ADJUSTING USING CucKoo SEARCH AlgORITHM}

The CS algorithm is a nature-inspired metaheuristic algorithm inspired in the obligate brood parasitism behavior of some cuckoo bird species which lay their own eggs in nests of other birds [30]-[32]. If host birds realize that a cuckoo laid an egg in their nests, they will either remove the egg from the nest or just abandon it and build a new one. In this optimization algorithm, each nest represents a potential solution [30], [31], [34].

In general terms, CS algorithm implementation can be done through three ideal rules [30], [31], [35], which are: 1) Each cuckoo lays one egg at a time and dumps it in a randomly chosen nest. Each egg represents a solution; 2) The best nests with high quality of eggs (i.e., better solutions) will carry over to the next generations; and, 3) The number of available host nests (i.e., number of solutions per generation) is fixed and the egg laid by a cuckoo is discovered by the host bird with a probability, pa $\in[0,1]$.

The CS algorithm involves two phases: At first, the initial random population is created and then, CS goes into an iterative process where two random walks, Lévy flights, and biased/selective random walk, are used to search for new solutions within the boundaries of the search space according to the problem restrictions. The CS algorithm selects the solution with a better evaluation of the cost function in the set of solutions generated by the CS iterative process [30], [31], [36]. A detailed description of the different phases of the algorithm and its application for the optimization an FLC is presented in [37], whereas the application of the CS algorithm to the FLC described in [20] is exposed next and summarized in Fig. 3. 


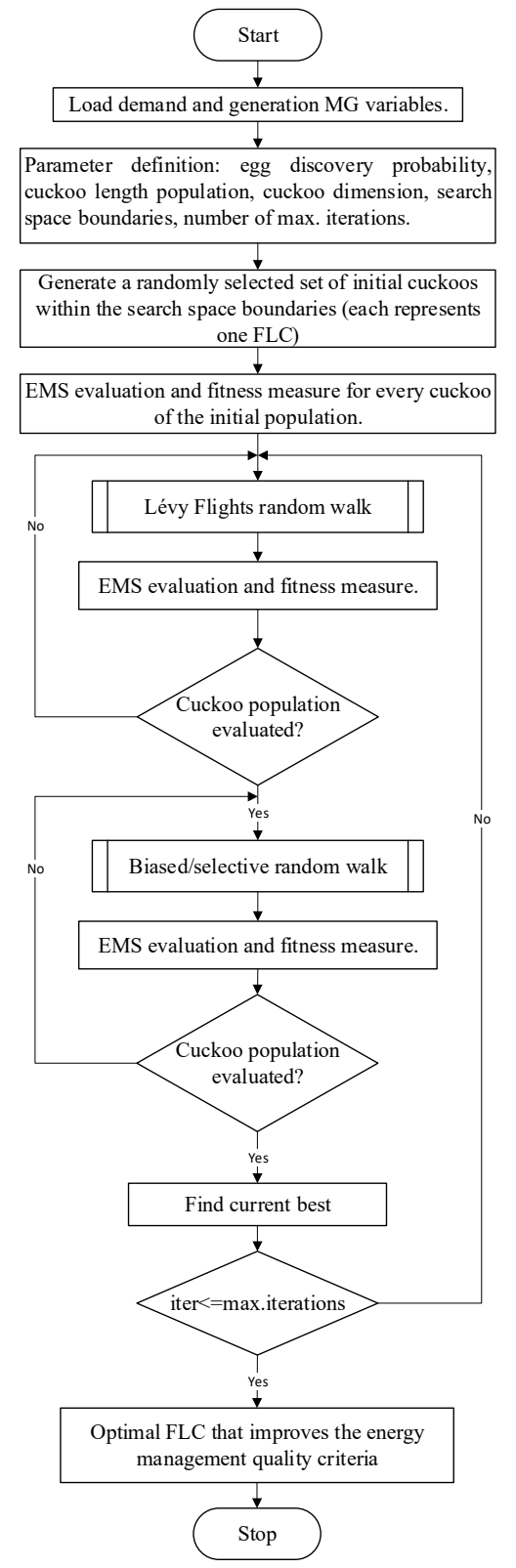

Fig. 3. Flowchart of the optimization process.

During the initialization, the CS algorithm creates a set of solutions which are randomly sampled from the search space. The dimension of the solution, $d$, is defined according to the total number of parameters to optimize. In accordance with the FLC described in [20], these parameters are related to the MF's mapping and the RB. In this regard, referring to Fig. 4 and Fig. 5, both inputs (i.e., $P_{E}$ and $S O C$ ) comprise five fuzzy sets and thirteen parameters (since every fuzzy set is represented by a triangular MF of three variable positions except for both located at the maximum and minimum universe of discourse limits that have one fixed positions and two variables) and output (i.e., $P_{F L C}$ ) comprises nine fuzzy sets with twenty-seven parameters, whereas the RB includes twenty-five parameters. Considering all these values, each solution is represented with $d=78$ parameters and the length of the population is fixed and with a value of $n=25$ [30]. Initial random solutions are generated separately for every $\mathrm{MF}$ and $\mathrm{RB}$ parameters, as follows:

$$
X_{G}=\left[X_{M F(G)}, X_{R B(G)}\right] \text {, }
$$

$$
\begin{gathered}
X_{M F(G)}=\left[M F_{A}+\delta_{A}, M F_{B}+\delta_{B}, M F_{C}+\delta_{C}\right], \\
X_{R B(G)}=\left[R B_{1}+\rho_{1}, R B_{2}+\rho_{2}, \cdots, R B_{25}+\rho_{25}\right],
\end{gathered}
$$

where $X_{G}$ is an initial random solution comprising of solutions for MF parameters and $\mathrm{RB}, X_{M F(G)}$ is a random initial solution for MF parameters at a first-generation $G$, $M F_{A}, M F_{B}, M F_{C}$ are typical triangular uniformly distributed MF's parameters, $\delta_{A, B, C} \sim(-5 \% \cdot R, 5 \% \cdot R)$ are random numbers drawn from a uniform distribution, $R$ is the variable range for every input/output variable (a $5 \%$ design value that has been defined since the EMS is sensible to small MF parameter variations), $X_{R B(G)}$ is a random initial solution for RB parameters at a first-generation $G, R B_{1}, R B_{2}, \ldots, R B_{25}$ are a set of initial rule base parameters defined according to the heuristic knowledge of the microgrid, and $\rho \sim N(0,1)$ is a random integer number drawn from a uniform distribution.

Once the initial random population is created, the CS algorithm evaluates every candidate solution, so that the best ones obtained pass through the next generations. It is worth pointing out that, during a minimization/maximization process, the 'selection of best' step results crucial, so that, the best solution according to problem's objectives of optimization can be obtained at the end of the algorithm.

After the initialization and with the information that possible good solutions were obtained in a previous generation, CS starts an intensification (local search) process so that better solutions can be obtained. CS intensification for MF parameters is carried out using a Lévy Flights random walk with a step-size drawn from a Lévy distribution that is characterized by its infinite mean and variance, enabling CS to explore the search space more efficiently than other algorithms that use a standard Gaussian process [30], [31].

At a generation $G>0$, Lévy Flights random walk is formulated as follows:

$$
\begin{gathered}
X_{M F(G+1)}=X_{M F(G)}+\alpha \cdot \operatorname{Lévy}(\beta), \\
\alpha=\alpha_{0} \cdot\left(X_{M F(G)}-X_{M F(\text { best })}\right),
\end{gathered}
$$

where $X_{M F(G+1)}$ is a candidate new solution, $X_{M F(G)}$ is a candidate solution selected from the cuckoo population, Lévy $(\beta)$ is a random step-size drawn from Lévy distribution calculated via the so-called Mantegna's algorithm for a symmetric Lévy stable distribution, $\alpha>0$ is a set of step-sizes that are related to the scale of the problem [30], [31], and $\alpha_{0} \sim U(0.01,0.1)$ is a varying scaling factor drawn from a uniform distribution [36].

The CS intensification for RB parameters are calculated as follows:

$$
X_{R B(G+1)}=X_{R B(G)}+s \cdot\left(X_{R B(G)}-X_{R B(\text { bets })}\right),
$$

where $X_{R B(\mathrm{G}+1)}$ is a candidate new solution for RB parameters obtained after intensification process, $X_{R B(\mathrm{G})}$ is a candidate solution at a generation $G$ selected from the cuckoo population, $s$ is vector of integer numbers drawn from a normal standard distribution $N(0,1)$ to displace the RB values, and $X_{R B \text { (best) }}$ is the best solution for RB parameters obtained so far in the iterative process and together with membership function's parameters, better optimizes the cost function. 
After a local search, global search is applied to find new solutions far enough from the current best solutions by using far-field randomization [31]. First, a trial solution is built using a mutation of the current solution and a differential step size from two stochastically selected solutions. Second, a new solution is generated by a crossover operator from the current and the trial solutions [36]. This procedure is formulated as follows:

$$
X_{G+1}=\left\{\begin{array}{ll}
X_{G}+r \cdot\left(X_{p(G)}-X_{q(G)}\right) & ; p_{a} \leq 0.25 \\
X_{G} & ; p_{a}>0.25
\end{array},\right.
$$

where $p$ and $q$ are random indexes so that $X_{p(G)}$ and $X_{q(G)}$ are randomly selected solutions, $r \sim U(0,1)$ is a random number drawn from a uniform distribution, and $p_{a}=0.25$ is the solution discovery rate [36], [38].

Both local and global searches are applied to every solution of the population and a solution is replaced by another one or kept for the next generations, according to the following:

$$
X_{G+1}= \begin{cases}X_{G+1} & ; \text { fitness }\left(X_{G+1}\right)<\text { fitness }\left(X_{G}\right) \\ X_{G} & ; \text { otherwise }\end{cases}
$$

where fitness is the cost function, and $X_{(G)}$ and $X_{(G+l)}$ are candidate solutions comprising of $X_{M F(G)}$ and $X_{M F(G+l)}$ and, $X_{R B(G)}$ and $X_{R B(G+I)}$, respectively. Furthermore, the update process of the $X_{\text {best }}$ in every generation $G$ is defined by:

$$
X_{\text {best }} \leftarrow \text { fitness }\left(X_{\text {bets }}\right)<\text { fitness }\left(X_{G}\right) \text {. }
$$

\section{SIMULATION AND COMPARISON RESULTS}

The real data from one year (2013-2014) provided by the MG installed at Public University of Navarre (UPNa) (Pamplona, Spain: 4249'06”N 1³8'39'O) are considered for simulation purposes. These data have been obtained through power analyzers recording the output power of both RES and load consumption with a sampling period of $15 \mathrm{~min}$ $\left(T_{S}=900 \mathrm{~s}\right)$. The FLC parameters adjustment is carried out through numerical simulations by means of Matlab $\AA$ in an Intel(R) Core (TM) i7-4510U CPU (2.00GHz) 12 GB RAM computer, where most of the computation time is spent for solving the 300 iterations for minimizing the cost function.

The processing time obviously depends on the processor speed and can be rather long since input data correspond to at least 3 vectors (wind and PV generation as well as load) of $(365$ days $\cdot 24 \mathrm{~h}) /($ day $\cdot 4$ samples $/ \mathrm{h})=35040$ samples $/$ vector and 300 iterations. However, it can be noted that almost all possible energy scenarios will be covered along a year. Moreover, the use of the CS algorithm leads to a simulation time reduction of around $33 \%$ compared with the previous heuristic trial and error procedure presented in [20].

Furthermore, a weighting factor of $w=2$ has been implemented in the cost function defined in (12) to prioritize the minimization of $P_{G, M A X}, P_{G, M I N}$, and $M P D$ over $A P D$, $P P V$, and $P V R$ since are dependent of the first three ones and because the main objectives of the EMS is to minimize the power ramp-rates, power peaks, and fluctuations in the power exchanged with the grid. The comparison of the fuzzy-based EMS (hereinafter referred to as H-FLC EMS) presented in [20] after passing through the described Cuckoo adjustment process, hereinafter referred to as CS-FLC EMS, is presented next. For comparison purposes, the reference values used in (13) and (14) correspond to the quality criteria values obtained by [20].

Fig. 4 and Fig. 5 show the MFs of inputs and output for both EMSs. As it can be seen, there are strong differences between them, especially when comparing the output MFs and its mapping. The variation range of the output variable has been reduced after the CS optimization process (i.e., from $-0.8 \mathrm{~kW} \leq P_{F L C} \leq 1.35 \mathrm{~kW}$ to $-0.5051 \mathrm{~kW} \leq P_{F L C} \leq$ $0.7863 \mathrm{~kW}$ ), which implies that the output of the fuzzy controller will have less variability and consequently the optimized strategy will achieve a smoother grid power profile.

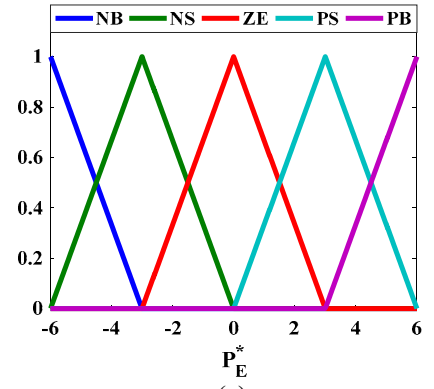

(a)

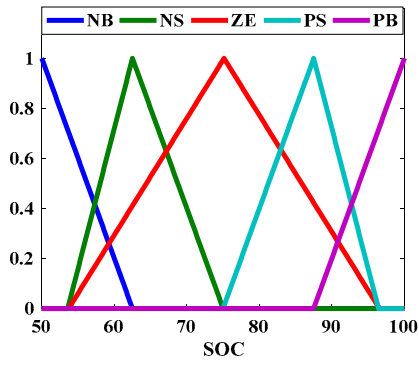

(b)

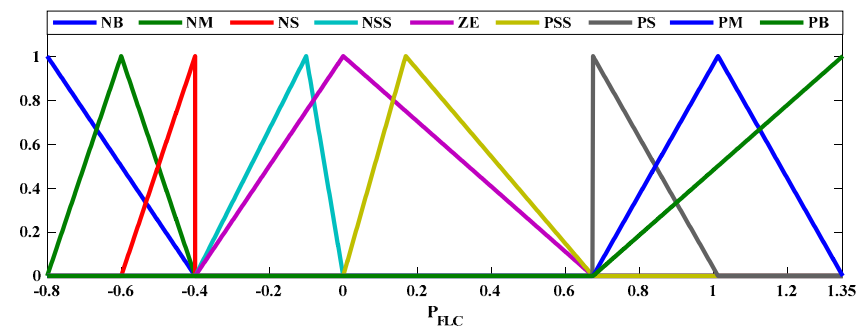

(c)

Fig. 4. Membership functions of the H-FLC EMS. (a) input $P_{E}^{*}$, (b) input SOC, and (c) output $P_{F L C}$.

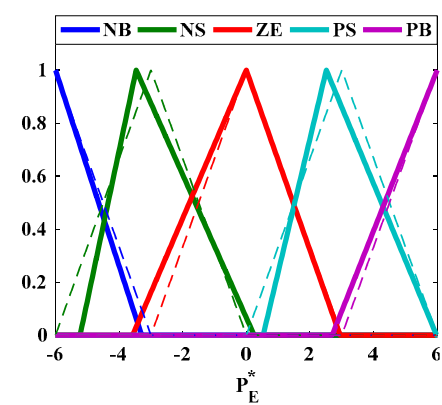

(a)

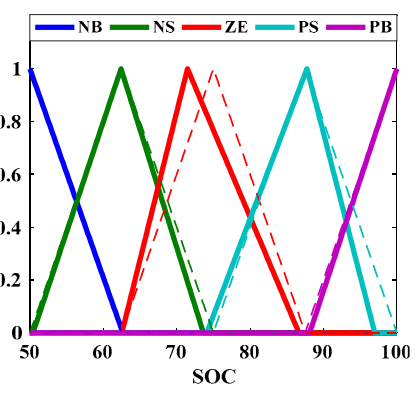

(b)

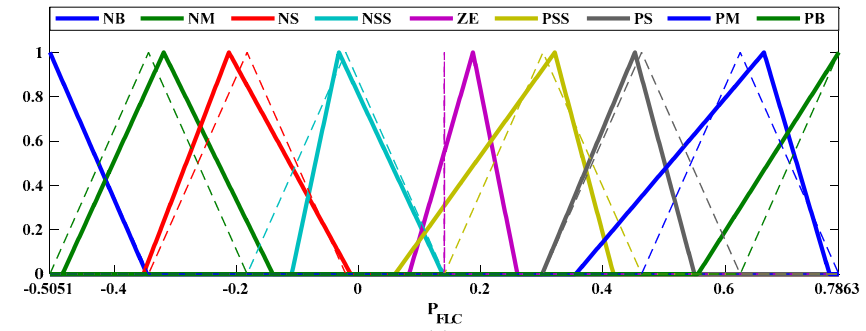

(c)

Fig. 5. Membership functions of the CS-FLC EMS. (a) input $P_{E}^{*}$, (b) input SOC, and (c) output $P_{F L C}$.

Similarly, when comparing the fuzzy RB of both EMSs, shown in TABLE I. and TABLE II. it can be noted that 13 
rules change (highlighted in cyan color) in the optimized rule base of the CS-FLC EMS.

TABLE I. Heuristic ApProACH Rule Base H-FLC EMS [20]

\begin{tabular}{|c|c|c|c|c|c|c|}
\hline \multirow{2}{*}{} & \multicolumn{5}{|c|}{$\boldsymbol{P}_{\boldsymbol{E} \text { FLC }}^{*}(\boldsymbol{n})$} \\
\cline { 3 - 8 } & & NB & NS & ZE & PS & PB \\
\hline \multirow{4}{*}{ SOC (n) } & NB & PB & PM & PS & PM & PB \\
\cline { 2 - 8 } & NS & PM & PS & PSS & PS & PM \\
\cline { 2 - 8 } & ZE & NS & ZE & ZE & PSS & NSS \\
\cline { 2 - 8 } & PS & NM & NS & NSS & NS & NM \\
\cline { 2 - 8 } & PB & NB & NSS & NM & NM & NB \\
\hline
\end{tabular}

TABLE II. OPTIMIZED RULE BASE THROUGH CUCKOO SEARCH ALGORITHM

\begin{tabular}{|c|c|c|c|c|c|c|}
\hline \multirow{2}{*}{} & \multicolumn{5}{|c|}{$\boldsymbol{P}_{\boldsymbol{E}}^{*}(\boldsymbol{n})$} \\
\cline { 3 - 7 } \multicolumn{2}{|c|}{} & NB & NS & ZE & PS & PB \\
\hline \multirow{4}{*}{ SOC (n) } & NB & ZE & ZE & PS & PM & PB \\
\cline { 2 - 7 } & NS & NSS & ZE & PSS & PS & PB \\
\cline { 2 - 8 } & ZE & NS & NSS & ZE & PSS & PS \\
\cline { 2 - 8 } & PS & NM & NS & NSS & ZE & PSS \\
\cline { 2 - 8 } & PB & NB & NM & NS & NS & ZE \\
\hline
\end{tabular}

Regarding the performance of the CS-FLC EMSs strategy, it can be seen in Fig. 6 that the optimized strategy minimizes the power peaks and fluctuations in the power exchanged with the grid achieving a maximum power delivered by the grid of $P_{G, M A X}=2.51 \mathrm{~kW}(2.56 \mathrm{~kW}$ for the $\mathrm{H}-\mathrm{FLC}$ EMS) and a maximum power injected to the grid of $P_{G, M I N}=-1.66 \mathrm{~kW}(-1.89 \mathrm{~kW}$ for the H-FLC EMS).

In addition, the proper behavior of the battery SOC evolution during the year under study achieved for both strategies can be evidenced in Fig. 7, where it can be seen that the battery SOC evolution reached by the CS-FLC EMS improves the one achieved by the H-FLC EMS. In short, the battery SOC of the optimized strategy is kept in a range between the $70 \%$ and $80 \%$ of the rated battery capacity during the $48.35 \%$ of the year under study, which leads to the ESS to be further prepared to compensate the fluctuations that may exist in the MG.

Finally, analyzing the resulting values of the defined quality criteria summarized in TABLE III. it can be verified that the optimized CS-FLC EMS achieves an important reduction in the quality criteria magnitude where there is a widespread reduction of $44.2 \%$ in the MPD criterion. As shown in Fig. 8, the grid power profile ramp-rates have been reduced in the year under study.

\section{CONCLUSIONS}

In this study, an FLC for an EMS of a grid-tied domestic electro-thermal microgrid obtained by means of CS algorithm is proposed and verified through simulations. To evaluate the performance of the proposed FLC, energy management quality criteria of the EMS during a one-year simulation is measured. In this regard, membership functions and rule-base parameters obtained using CS algorithm result in a higher energy management quality (Table III) in comparison with parameters obtained by means of heuristic trial and error procedures used in previous studies, minimizing $\mathrm{MG}$ power profile fluctuations and keeping battery SOC range within a secure range of operation.

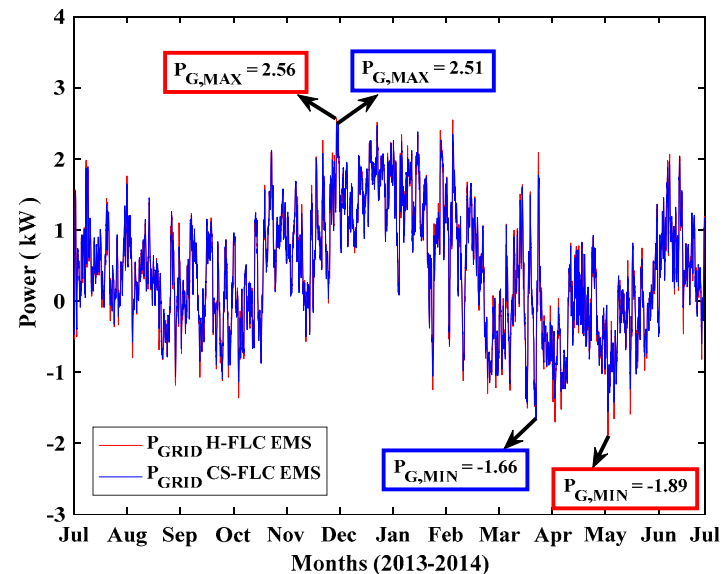

Fig. 6. Grid power profile comparison.

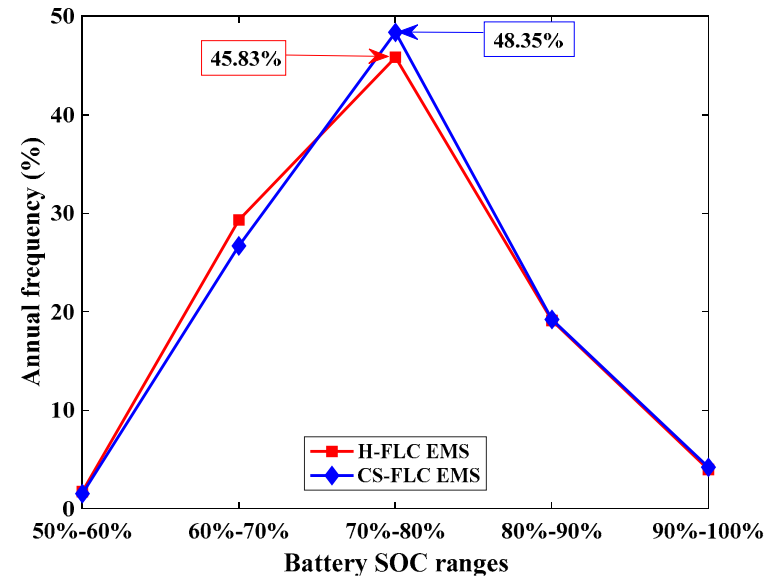

Fig. 7. Histogram of battery SOC ranges comparison.

TABLE III. QUALITY CRITERIA COMPARISON

\begin{tabular}{|c|c|c|c|c|c|c|}
\hline \multirow{2}{*}{$\begin{array}{c}\text { EMS } \\
\text { Strategy }\end{array}$} & $\begin{array}{c}\text { Grid Power Profile Quality Criteria } \\
(\mathbf{P W})\end{array}$ & $\begin{array}{c}\boldsymbol{P}_{G, M I N} \\
(\mathbf{k W})\end{array}$ & $\begin{array}{c}\boldsymbol{M P D} \\
(\mathbf{W} / \mathbf{h})\end{array}$ & $\begin{array}{c}\boldsymbol{A P D} \\
(\mathbf{W} / \mathbf{h})\end{array}$ & $\boldsymbol{P P V}$ & $\boldsymbol{P V R}$ \\
\hline EMS [20] & 2.56 & -1.89 & 846 & 75 & 1.26 & 0.34 \\
\hline $\begin{array}{c}\text { CS } \\
\text { optimized } \\
\text { EMS }\end{array}$ & 2.51 & -1.66 & 472 & 75 & 1.25 & 0.32 \\
\hline
\end{tabular}

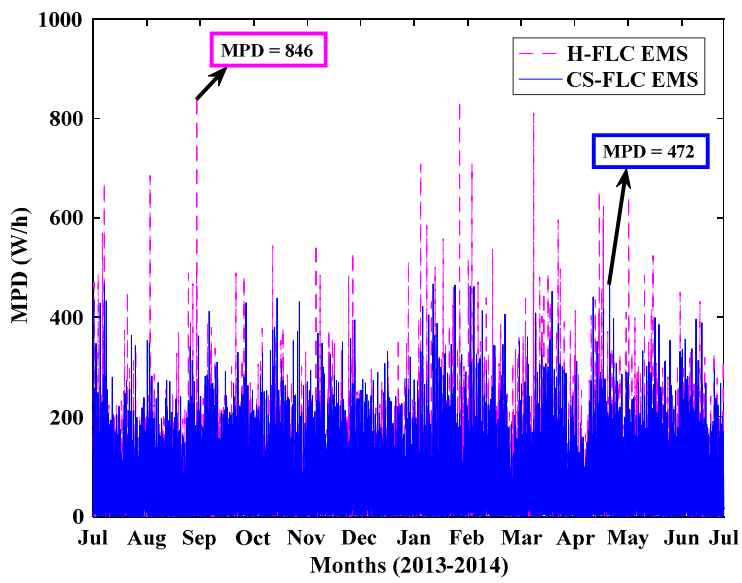

Fig. 8. Grid power ramp-rate comparison. 


\section{ACKNOWLEDGMENT}

This work is part of the projects 2019-PIC-003-CTE from the Research Group of Propagation, Electronic Control, and Networking (PROCONET) of Universidad de las Fuerzas Armadas ESPE and Project I+D from Government of Navarra, and Hybridization of advanced electrical storage technologies based on renewable energies DPI2016-08642-R (HIBRITAER). This work has been partially supported by the Spanish Ministry of Industry and Competitiveness under the grants DPI2017-85404, DPI2015-67292-R, and FEDER ECO 1823-2015.

\section{REFERENCES}

[1] International Energy Agency, "Key world energy statistics," 2018.

[2] International Energy Agency, CO2 Emissions from Fuel Combustion 2018. OECD, 2018.

[3] H. Kanchev, D. Lu, F. Colas, V. Lazarov, and B. Francois, "Energy Management and Operational Planning of a Microgrid With a PVBased Active Generator for Smart Grid Applications," IEEE Trans. Ind. Electron., vol. 58, no. 10, pp. 4583-4592, Oct. 2011.

[4] R. H. Lasseter, "MicroGrids," in Proc. Power Eng. Soc. Winter Meeting, New York, NY, USA, Jan. 2002, vol. 1, pp. 305-308.

[5] N. Hatziargyriou, H. Asano, R. Iravani, and C. Marnay, "Microgrids," IEEE Power and Energy Mag., vol. 5, no. 4, pp. 78-94, Jul. 2007.

[6] F. Katiraei, R. Iravani, N. Hatziargyriou, and A. Dimeas, "Microgrids management," IEEE Power and Energy Mag., vol. 6, no. 3, pp. 5465, May. 2008.

[7] E. Unamuno and J. A. Barrena, "Hybrid ac/dc microgrids—Part I: Review and classification of topologies," Renewable and Sustainable Energy Reviews, vol. 52, pp. 1251-1259, Dec. 2015.

[8] M. Soshinskaya, W. H. J. Crijns-Graus, J. M. Guerrero, and J. C. Vasquez, "Microgrids: Experiences, barriers and success factors," Renewable and Sustainable Energy Reviews, vol. 40, pp. 659-672, Dec. 2014.

[9] H. Bevrani, B. Francois, and T. Ise, "Microgrid Planning and Energy Management," in Microgrid Dynamics and Control, Hoboken, NJ, USA: John Wiley \& Sons, Inc., Jul. 2017, pp. 589-661.

[10] A. Hirsch, Y. Parag, and J. Guerrero, "Microgrids: A review of technologies, key drivers, and outstanding issues," Renewable and Sustainable Energy Reviews, vol. 90, pp. 402-411, Jul. 2018.

[11] D. E. Olivares et al., "Trends in microgrid control," IEEE Trans. Smart Grid, vol. 5, no. 4, pp. 1905-1919, Jul. 2014.

[12] M. H. Athari and M. M. Ardehali, "Operational performance of energy storage as function of electricity prices for on-grid hybrid renewable energy system by optimized fuzzy logic controller," Renewable Energy, vol. 85, pp. 890-902, Jan. 2016.

[13] C. Zhang, Y. Xu, Z. Y. Dong, and K. P. Wong, "Robust Coordination of Distributed Generation and Price-Based Demand Response in Microgrids," IEEE Trans. Smart Grid, vol. 9, no. 5, pp. 4236-4247, Sep. 2018.

[14] D. Arcos-Aviles et al., "A Review of Fuzzy-Based Residential GridConnected Microgrid Energy Management Strategies for Grid Power Profile Smoothing," in Energy Sustainability in Built and Urban Environments, 1st ed., T.-E. Motoasca, A. K. Agarwal, and H. Breesch, Eds. Springer Nature, Jan. 2019, pp. 167-200.

[15] J. Pascual, P. Sanchis, and L. Marroyo, "Implementation and Control of a Residential Electrothermal Microgrid Based on Renewable Energies, a Hybrid Storage System and Demand Side Management," Energies, vol. 7, no. 1, pp. 210-237, Jan. 2014.

[16] J. Pascual, J. Barricarte, P. Sanchis, and L. Marroyo, "Energy management strategy for a renewable-based residential microgrid with generation and demand forecasting," Applied Energy, vol. 158, pp. 12-25, Nov. 2015.

[17] J. Wu, X. Xing, X. Liu, J. M. Guerrero, and Z. Chen, "Energy Management Strategy for Grid-Tied Microgrids Considering the Energy Storage Efficiency," IEEE Trans. Ind. Electron., vol. 65, no. 12, pp. 9539-9549, Dec. 2018.

[18] D. Arcos-Aviles, J. Pascual, L. Marroyo, P. Sanchis, and F. Guinjoan, "Fuzzy Logic-Based Energy Management System Design for Residential Grid-Connected Microgrids," IEEE Trans. Smart Grid, vol. 9, no. 2, pp. 530-543, Mar. 2018.
[19] D. Arcos-Aviles, J. Pascual, F. Guinjoan, L. Marroyo, P. Sanchis, and M. P. Marietta, "Low complexity energy management strategy for grid profile smoothing of a residential grid-connected microgrid using generation and demand forecasting," Applied Energy, vol. 205, pp. 69-84, Nov. 2017.

[20] D. Arcos-Aviles et al., "Fuzzy-based energy management of a residential electro-thermal microgrid based on power forecasting," in IECON 2018 - 44th Annual Conference of the IEEE Industrial Electronics Society, Washington, DC, USA, Oct. 2018, pp. 18241829 .

[21] D. Arcos-Aviles, F. Guinjoan, M. P. Marietta, J. Pascual, L. Marroyo, and P. Sanchis, "Energy management strategy for a grid-tied residential microgrid based on Fuzzy Logic and power forecasting," in IECON 2016 - 42nd Annual Conference of the IEEE Industrial Electronics Society, Florence, Italy, Oct. 2016, pp. 4103-4108.

[22] D. Arcos-Aviles et al., "Fuzzy energy management strategy based on microgrid energy rate-of-change applied to an electro-thermal residential microgrid," in 2017 IEEE 26th International Symposium on Industrial Electronics (ISIE), Edinburgh, UK, Jun. 2017, pp. 99105.

[23] D. A. Aviles, F. Guinjoan, J. Barricarte, L. Marroyo, P. Sanchis, and H. Valderrama, "Battery management fuzzy control for a grid-tied microgrid with renewable generation," in IECON 2012 - 38th Annual Conference on IEEE Industrial Electronics Society, Montreal, QC, Canada, Oct. 2012, pp. 5607-5612.

[24] M. Taruwona and C. N. Nyirenda, "Particle Swarm Optimization of a Mamdani Fuzzy Logic Based Charge Controller for Energy Storage Systems," in IEEE 2018 Open Innovations Conference (OI), Johannesburg, South Africa, Oct. 2018, pp. 73-78.

[25] S. M. Abd Elazim and E. S. Ali, "Optimal Power System Stabilizers design via Cuckoo Search algorithm," International Journal of Electrical Power \& Energy Systems, vol. 75, pp. 99-107, Feb. 2016.

[26] A. Y. Abdelaziz and E. S. Ali, "Cuckoo Search algorithm based load frequency controller design for nonlinear interconnected power system," International Journal of Electrical Power \& Energy Systems, vol. 73, pp. 632-643, Dec. 2015.

[27] S. Berrazouane and K. Mohammedi, "Parameter optimization via cuckoo optimization algorithm of fuzzy controller for energy management of a hybrid power system," Energy Conversion and Management, vol. 78, pp. 652-660, Feb. 2014.

[28] P. Civicioglu and E. Besdok, "A conceptual comparison of the Cuckoo-search, particle swarm optimization, differential evolution and artificial bee colony algorithms," Artif. Intell. Rev., vol. 39, no. 4, pp. 315-346, Apr. 2013.

[29] T. Wang, M. Meskin, and I. Grinberg, "Comparison between particle swarm optimization and Cuckoo Search method for optimization in unbalanced active distribution system," in 2017 IEEE International Conference on Smart Energy Grid Engineering (SEGE), Oshawa, ON, Canada, Aug. 2017, pp. 14-19.

[30] X. S. Yang, Nature-inspired optimization algorithms, 1st ed. Amsterdam, Nederlands: Elsevier, 2014

[31] X.-S. Yang and Suash Deb, "Cuckoo Search via Lévy flights," in 2009 World Congress on Nature \& Biologically Inspired Computing (NaBIC), Coimbatore, India, Dec. 2009, pp. 210-214.

[32] R. Rajabioun, "Cuckoo Optimization Algorithm," Applied Soft Computing, vol. 11, no. 8, pp. 5508-5518, Dec. 2011.

[33] R. T. Marler and J. S. Arora, "The weighted sum method for multiobjective optimization: new insights," Struct. Multidisc. Optim., vol. 41, no. 6, pp. 853-862, Jun. 2010.

[34] R. B. Payne and M. D. Sorenson, The cuckoos. New York, EEUU: Oxford University Press, 2005.

[35] K. N. Abdul Rani et al., "Modified cuckoo search algorithm in weighted sum optimization for linear antenna array synthesis," in 2012 IEEE Symposium on Wireless Technology and Applications (ISWTA), Bandung, Indonesia, Sep. 2012, pp. 210-215.

[36] L. Wang, Y. Yin, and Y. Zhong, "Cuckoo search with varied scaling factor," Front. Comput. Sci., vol. 9, no. 4, pp. 623-635, Aug. 2015.

[37] G. García-Gutiérrez et al., "Fuzzy Logic Controller Parameter Optimization Using Metaheuristic Cuckoo Search Algorithm for a Magnetic Levitation System," Appl. Sci., vol. 9, no. 12, p. 2458, Jun. 2019.

[38] I. Fister, D. Fister, and I. Fister, "A comprehensive review of cuckoo search: variants and hybrids," Int. J. Math. Model. Numer. Optim., vol. 4, no. 4, p. 387, 2013. 